\title{
Analysis on the Medication Rule of Famous Old TCM Doctors in the Treatment of Cervical Spondylosis Based on Data Mining
}

\author{
Huaijing Wang, Zhongyi Zhu, Ping Wang*, Bifeng Fu \\ First Teaching Hospital of Tianjin Universality of Traditional Chinese Medicine and National Clinical Research Center for Chinese \\ Medicine Acupuncture and Moxibustion, Tianjin 300193, China \\ Email: 2390352180@qq.com
}

\begin{abstract}
Purpose - To use data mining methods to study the medication rules of famous and old traditional Chinese medicine (TCM) doctors in the treatment of cervical spondylosis. Methods - To search and collate the experience books of Chinese medicine masters, published journal literature and shared medical cases of national famous TCM studios, screen the data of famous TCM treatment of cervical spondylosis, standardize the medical case database, standardize the name of medicine, analyze the medication rules of traditional Chinese medicine by using frequency analysis, cluster analysis and association rule analysis, and obtain the medication frequency and syndrome treatment points Cloth, medicine, core prescription, etc. Results - Among the 199 prescriptions collected, 218 Chinese medicines were involved, with a total frequency of 2461. Among them, 33 Chinese medicines are with a frequency of more than or equal to 20. Glycyrrhiza uralensis, Angelica sinensis, Ligusticum chuanxiong, Pueraria lobata, Radix Paeoniae Alba, astragalus, Poria cocos, Rhizoma Notopterygii and Carthamus tinctorius ranked in the top 10, and the drugs of Invigorating Qi and activating blood circulation had the highest frequency. There are 7 kinds of drugs in cluster analysis, 10 drug pair association rules and 60 drug group association rules. Conclusion - In the treatment of cervical spondylosis with traditional Chinese medicine, the famous old TCM doctor mainly uses the methods of Invigorating Qi and promoting blood circulation, invigorating liver and kidney, eliminating wind and dampness, resolving phlegm and dredging collaterals, which further proves that the main etiology and pathogenesis of cervical spondylosis is deficiency and excess. In the treatment, we should pay attention to invigorating Qi and nourishing blood, and taking into account cold and dampness.
\end{abstract}

Keywords: data mining, cervical spondylosis, famous old TCM doctor, medication rule

Cervical spondylosis is caused by wind-cold, trauma, strain and improper posture for a long time, resulting in obstruction of Qi and blood. It belongs to the category of "arthralgia" in Chinese medicine ${ }^{[1]}$. The main clinical manifestations are neck pain, numbness of the limbs, headache, dizziness, hyperhidrosis and other symptoms. In severe cases, there are performances such as unstable walking and neck and shoulder muscle atrophy. The pathological mechanism of cervical spondylosis is degeneration of cervical intervertebral discs, joints, and ligaments ${ }^{[2]}$. Recent epidemiology shows that: cervical spondylosis ranks second among the top ten chronic diseases in the world ${ }^{[3]}$; there are more than 900 million patients with cervical spondylosis worldwide, and my country accounts for $17.3 \%$ of the number of patients, about 160 million ${ }^{[4]}$. At present, there is no specific treatment, mainly through anti-inflammatory analgesics, physical therapy, surgery and other symptomatic treatments. However, the treatment methods are limited and single, with many adverse drug reactions and high prices, which increase the economic and psychological burden of patients. In recent years, traditional Chinese medicine treatment of cervical spondylosis has achieved satisfactory results in improving the condition, reducing adverse drug reactions, and improving the quality of life of patients ${ }^{[5]}$. The well-known old Chinese doctor has remarkable curative effect in treating cervical spondylosis, and has extremely high clinical guiding significance. However, the existing research only focuses on the medication rule of a certain old Chinese doctor, and there is a lack of research on multiple old Chinese doctors ${ }^{[6]}$. This study seeks common ground, summarizes and sorts out the literature and series of famous and old traditional Chinese medicine prescriptions for treatment of cervical spondylosis, and excavates the laws of medication, syndrome and treatment. This study uses methods such as frequency analysis, cluster analysis, association rule analysis, and complex network analysis to excavate the prescription rules of well-known old Chinese medicine practitioners in the treatment of cervical spondylosis, and provide reference value for clinical practice. 


\section{Materials and methods}

\subsection{Data source search}

Routinely search databases such as CNKI, Wanfang Database, Weipu Database, Chaoxing Library, etc., with manual search. The search keywords are "Master of Traditional Chinese Medicine", "Famous Chinese Medicine", "Cervical Spondylopathy" and "Xiang Bi". Two researchers independently searched and screened prescriptions based on inclusion and exclusion criteria. After completion, cross-check, and if there are differences, they will be resolved through discussion and negotiation. If there is a serious disagreement that cannot be resolved through negotiation, the third investigator and the research supervisor will decide whether to include it.

\subsection{Inclusion and exclusion criteria}

Inclusion criteria: (1) The selected famous old Chinese medicine records must come from Chinese medicine masters and famous Chinese medicine doctors above the provincial level. The judgment standards for Chinese medicine masters and famous Chinese medicine doctors refer to the high-ranking, respected, and respected documents selected by the Ministry of Human Resources and Social Security and the State Administration of Traditional Chinese Medicine in 2009. Famous doctors with superb medical skills; (2) The treatment target is clearly diagnosed as cervical spondylosis or Xiangbi; (3) The medical records with complete medical records, complete prescriptions and clear curative effects; Take the basic prescription, and the prescription is taken orally.

Exclusion criteria: (1) Medical records that did not list specific drug names, only the names of prescriptions; (2) Medical records where prescriptions were not taken internally; (3) Medical records collected repeatedly; (4) Medical records where acupuncture, manipulation, traction or physical therapy were the main treatment (5) Medical records of patients with other diseases as the main disease, and on this basis, the symptoms of cervical spondylosis and cervical spondylosis secondary to it.

\subsection{The establishment and organization of the database}

According to the above-mentioned standards, the prescriptions of famous and old Chinese medicine doctors that meet the standards are screened out, and relevant information about the prescriptions of medical records is collected. Enter the prescription source, drug composition, TCM syndrome type, treatment method, etc. into WPS Office Excel, and then use the third person to refer to the National Higher TCM School Planning Textbook (Ninth Edition) "Chinese Materia Medica" [7] to conduct a review of the entered TCM specification. If the medicine is not included in the textbook, it shall be regulated in accordance with the 2015 edition of "The Pharmacopoeia of the People's Republic of China"[8] and local provincial standards for Chinese medicinal materials. Some Chinese medicines with standardized names are shown in Table 1. TCM syndrome types and treatment methods refer to "Diagnostics of Traditional Chinese Medicine" ${ }^{\text {[9] }}$ and "Studies of TCM Muscles and Injuries" 2012 edition ${ }^{[10]}$. If there are syndrome types and treatment methods that are not found in the above books, please refer to the diagnostic criteria of cervical spondylosis in the previous edition of "Traditional Chinese Orthopedics and Traumatology". Part of the syndrome types that require standardized names are shown in Table 2, and the treatment methods that require standardized names are shown in Table 3. In the process of inputting syndrome types and treatment methods, attention should be paid to the following points. (1) If a group of prescriptions and treatments are compound pathogenesis, enter the standardized syndrome types one by one. (2) If the overall prescription is not mentioned, only mention And some drug treatments are not entered. (3) For the symptoms of cervical spondylosis corresponding to the symptoms, pain relief and muscle relaxation are not entered.

Table 1. Drugs with standardized names

\begin{tabular}{|c|c|c|c|}
\hline Original name & Rectification of name & Rectification of name & Original name \\
\hline Liquor Rhubarb & Rhubarb & Poria cocos & Poria \\
\hline Anhydrous sodium sulfate & Mirabilite & Pilose antler & $\begin{array}{l}\text { Antler glue, cornu cervi degelatinatum, } \\
\text { antler }\end{array}$ \\
\hline Angelica body, Angelica tail & Angelica sinensis & Radix astragali & $\begin{array}{l}\text { Astragalus root, Radix Astragali } \\
\text { preparata }\end{array}$ \\
\hline $\begin{array}{c}\text { Licorice roots northwest origin, baked } \\
\text { licorice }\end{array}$ & Liquorice & The root of kudzu vine & Pueraria thomsonii Benth \\
\hline Fructus corni, liquor cornus & Cornus officinalis & Dried old orange peel & Orange peel, dried tangerine peel \\
\hline
\end{tabular}


Table 2. Types of certificates that need to be standardized

\begin{tabular}{cc}
\hline Original certificate type & Standard certificate type \\
\hline Deficiency of liver-yin and kidney-yin; deficiency of liver and kidney & Liver and kidney deficiency \\
Weficiency of spleen and kidney; spleen-kidney Qi deficiency; spleen- \\
kidney yang deficiency \\
Phlegm-blood stasis \\
Cold and dampness invasion; cold-dampness block; cold dampness stagnate
\end{tabular}

Table 3. The methods of treatment that need to standardize names

\begin{tabular}{cc}
\hline \multicolumn{1}{c}{ The original treatment } & The normative treatment \\
\hline $\begin{array}{c}\text { Activating blood to remove blood stasis, regulating Qi and relieving pain; } \\
\text { activating blood, promoting Qi and relieving pain }\end{array}$ & Promoting blood circulation \\
$\begin{array}{c}\text { Dispelling cold and relieving pain; dispelling cold and relieving pain; } \\
\text { dispelling wind and cold and dredging collaterals }\end{array}$ & Expelling wind and removing cold \\
$\begin{array}{c}\text { Nourishing the kidney and liver; nourishing the kidney and strengthening } \\
\text { the supervision; nourishing the liver and nourishing the blood }\end{array}$ & Nourishing the liver and nourishing the kidney \\
$\begin{array}{c}\text { Resolving phlegm and promoting blood circulation; dissipating phlegm and } \\
\text { dissipating blood }\end{array}$ & Phlegm resolving and blood stasis dissipating \\
$\begin{array}{c}\text { Regulating Qi and nourishing blood; regulating Qi and nourishing blood; } \\
\text { regulating Qi and nourishing blood }\end{array}$ & Reinforcing Qi and nourishing blood \\
$\begin{array}{c}\text { Suppressing the liver-yang; suppressing the liver and suppressing yang; } \\
\text { suppressing the liver and dredging collaterals }\end{array}$ & Suppressing the liver and suppressing yang \\
\hline
\end{tabular}

\subsection{Database preprocessing and entry}

Assign the value of Chinese medicine in the database according to yes $=1$ and no $=0$, and make each Chinese medicine as a two-category statistical variable, enter it item by item in WPS Office Excel, and then perform classification and statistical analysis.

\subsection{Data analysis}

Use data mining methods to analyze the medication rules of well-known Chinese medicine practitioners in the treatment of cervical spondylosis. Use IBM SPSS Statistics 23.0 for database descriptive analysis and cluster analysis; use IBM SPSS Modeler 18.0 for database association rule analysis and complex network analysis.

\section{Results}

\subsection{Include prescription results}

According to the acceptance criteria, Shi Qi, Wei Guikang, Zhu Liangchun, Sun Shuchun, Liu Boling, Li Zhenhua, Zhou Zhongying, Mei Guoqiang, Wan Yousheng, Shi Yangshan and others were finally included in the treatment of cervical spondylosis, a total of 199 prescriptions. 52 prescriptions came from masters of traditional Chinese medicine, including Shi Qi, Wei Guikang, Zhu Liangchun, Sun Shuchun, Liu Boling, Li Zhenhua, Zhou Zhongying, Xiong Jibai, Mei Guoqiang, Ren Jixue, Li Ke, Wan Yousheng, Shi Yangshan, Shan Jianmin, Lin Rugao , Li Jinyong, and Liu Zuyi’s prescriptions are taken orally.

\subsection{Frequency analysis 2.2.1 Statistics of drug frequency}

In the medical records for the treatment of cervical spondylosis, the frequency of all drugs is counted, and the drugs with frequency $\geq 20$ are ranked in descending order. A total of 33 commonly used Chinese medicines with a total of 1531 frequencies are obtained. The frequency calculation formula: frequency/total frequency, see Table 4 . Tonic drugs, activating blood circulation and removing blood stasis drugs, anti-surgical drugs, and calming liver and extinguishing wind drugs are the main drugs. See Table 5 for specific drugs. 
Table 4. Frequency statistics of traditional Chinese medicine for cervical spondylosis

\begin{tabular}{|c|c|c|c|c|c|}
\hline $\begin{array}{c}\text { Traditional Chinese } \\
\text { medicine }\end{array}$ & $\begin{array}{c}\text { Frequency of } \\
\text { occurrence }\end{array}$ & Frequency $(\%)$ & $\begin{array}{l}\text { Traditional Chinese } \\
\text { medicine }\end{array}$ & $\begin{array}{c}\text { Frequency of } \\
\text { occurrence }\end{array}$ & Frequency $(\%)$ \\
\hline Licorice & 124 & 5.04 & Salvia & 38 & 1.54 \\
\hline Angelica & 115 & 4.67 & $\begin{array}{c}\text { Divaricate saposhnikovia } \\
\text { root }\end{array}$ & 34 & 1.38 \\
\hline Ligusticum wallichii & 102 & 4.14 & Jujube & 34 & 1.38 \\
\hline Pueraria & 95 & 3.86 & Ginger & 33 & 1.34 \\
\hline White peony & 93 & 3.78 & Rehmannia glutinosa & 28 & 1.14 \\
\hline Astragalus & 77 & 3.13 & Bupleurum & 25 & 1.02 \\
\hline Cassia twig & 71 & 2.89 & Corydalis & 25 & 1.02 \\
\hline Poria & 57 & 2.32 & Medicinal cyathula root & 25 & 1.02 \\
\hline Notopterygium root & 53 & 2.15 & Lumbricus & 24 & 0.97 \\
\hline Safflower & 46 & 1.87 & Codonopsis & 23 & 0.93 \\
\hline Atractylodes & 46 & 1.87 & Uncaria & 22 & 0.89 \\
\hline Tangerine peel & 44 & 1.79 & Caulis spatholobi & 22 & 0.89 \\
\hline Rehmannia glutinosa & 43 & 1.75 & Chinese clematis root & 21 & 0.85 \\
\hline Red peony & 42 & 1.71 & $\begin{array}{l}\text { Radix angelicae } \\
\text { pubescentis }\end{array}$ & 20 & 0.81 \\
\hline Pinellia & 41 & 1.67 & Cornus & 20 & 0.81 \\
\hline Gastrodia & 40 & 1.63 & Antler & 20 & 0.81 \\
\hline Peach kernel & 38 & 1.54 & & & \\
\hline
\end{tabular}

Table 5. Classification statistics of traditional Chinese medicines for cervical spondylosis

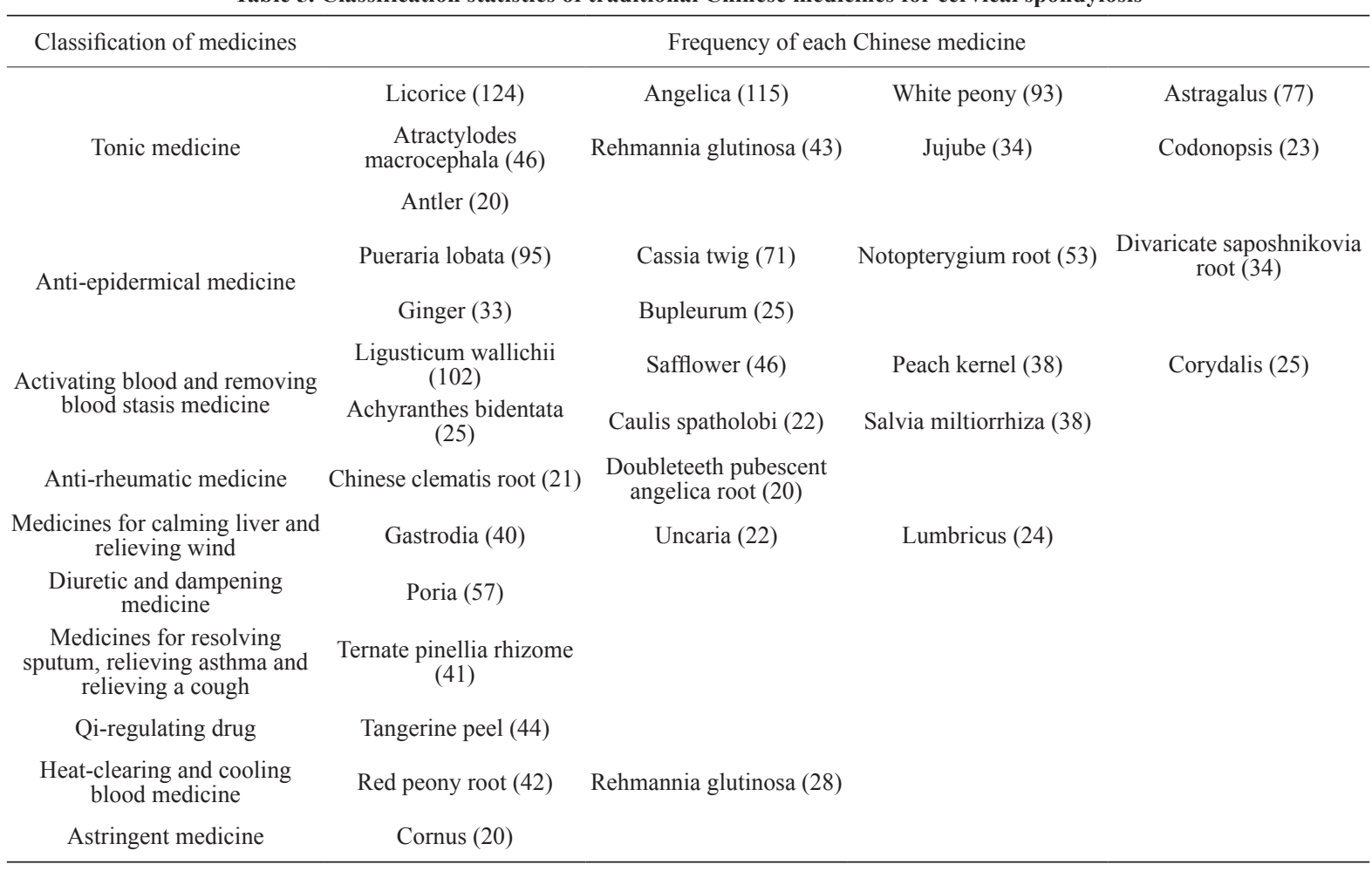

\subsubsection{Statistical results of syndrome types and frequency of treatment}

Statistics of cervical spondylosis syndrome types in the medical records of cervical spondylosis were counted. There 
were a total of 252 frequency, and there were 9 syndrome types with frequency $\geq 10$. The specific statistical results are shown in Table 6. The treatment of cervical spondylosis in the medical records of cervical spondylosis was counted. There were a total of 250 frequency and 8 treatment methods with frequency $\geq 10$. The specific statistical results are shown in Table 7 .

\begin{tabular}{ccc} 
& Table 6. Statistics of syndrome types & Frequency (\%) \\
\hline Types of syndrome & Frequency of occurrence & 15.8 \\
\hline Qi stagnation and blood stasis & 40 & 12.3 \\
Wind-cold paralysis & 31 & 11.9 \\
Insufficient liver and kidney & 30 & 6.7 \\
Rheumatic resistance & 17 & 6.3 \\
Deficiency of Qi and Blood & 16 & 6.0 \\
Qi deficiency and blood stasis & 15 & 14 \\
Phlegm and blood stasis obstruction & 13 & 5.6 \\
Hyperactivity of liver yang & 12 & 4.8 \\
\hline Resistance to phlegm and dampness & & 5.1 \\
\hline
\end{tabular}

Table 7. Statistics results of the methods of treatment

\begin{tabular}{ccc}
\hline The methods of treatment & Frequency of occurrence & Frequency (\%) \\
\hline Activating blood and promoting Qi & 67 & 26.8 \\
Dispel wind and cold & 54 & 21.6 \\
Nourishing liver and kidney & 42 & 16.8 \\
Dispel wind and dehumidification & 29 & 11.6 \\
Nourishing Qi and nourishing blood & 25 & 10.0 \\
Resolve phlegm and disperse blood stasis & 19 & 7.6 \\
Calm the liver and suppress the Yang & 13 & 5.2 \\
Dehumidification and phlegm removal & 11 & 4.4 \\
\hline
\end{tabular}

\subsection{Statistical results of meridian distribution of drug properties and flavors}

After statistics on the nature and flavor of drugs with a frequency of $\geq 20$, it is found that the prescription drugs for cervical spondylosis by masters of traditional Chinese medicine and famous Chinese medicine are mainly warm, lukewarm, and flat. The taste of the medicine is the most sweet, followed by bitter, pungent, and normal. The spleen meridian is the first, followed by the liver and lung meridians. See Figure 1-3.

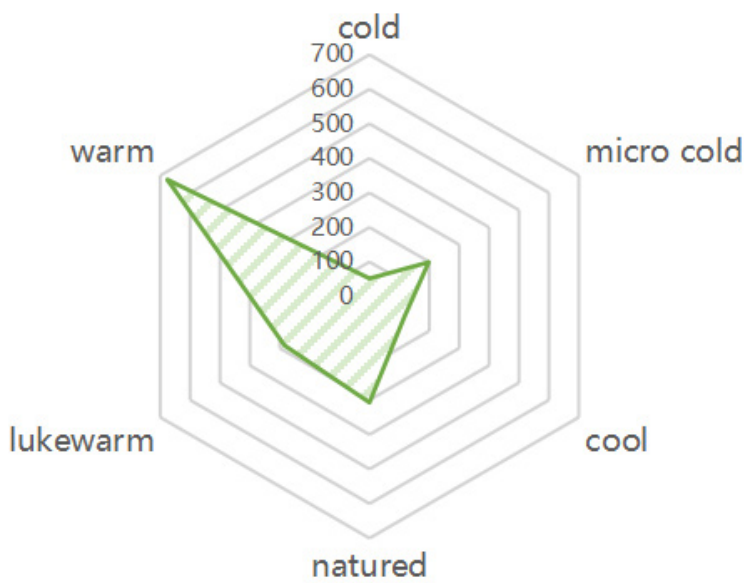

Figure 1. Radar chart of the four gasses of medicines

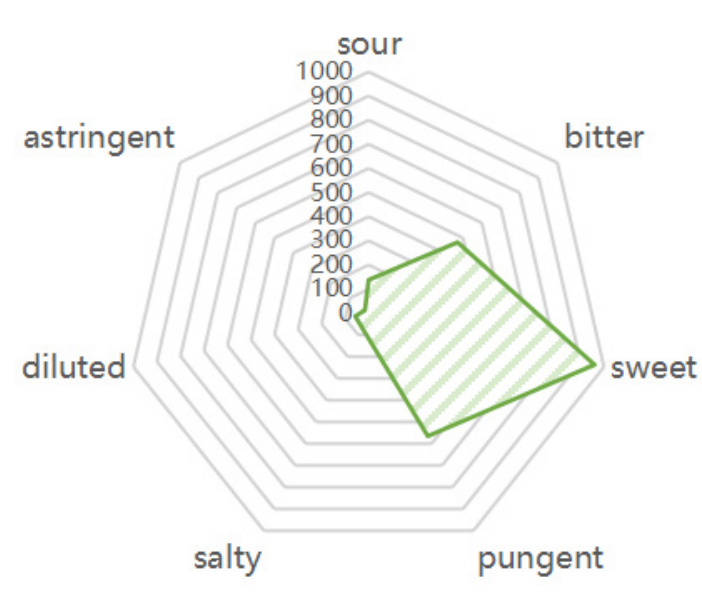

Figure 2. Radar chart of the five flavors of medicines 


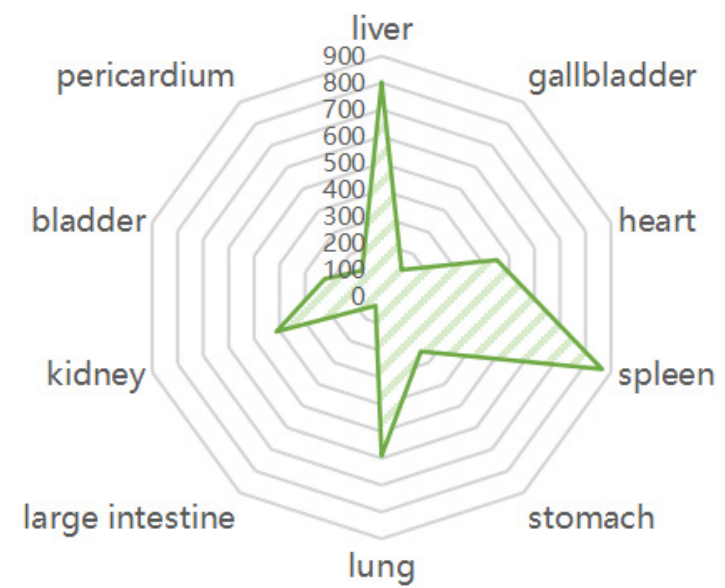

Figure 3. Radar chart of medicines' return

\subsection{The statistical results of cluster analysis of drugs with frequency $\geq \mathbf{2 0}$}

From the tree diagram in Figure 4, it can be observed that when the clustering interval is 20, drugs can be divided into seven categories. The composition, source, and function of each category of drugs are shown in Table 8.

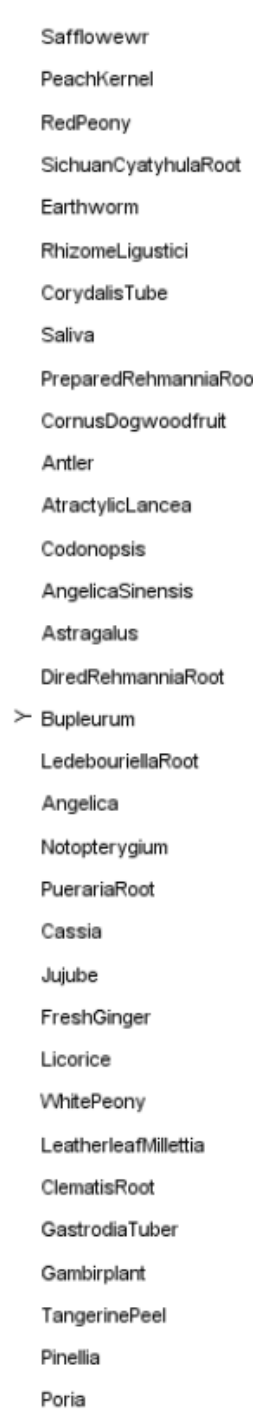

Poria

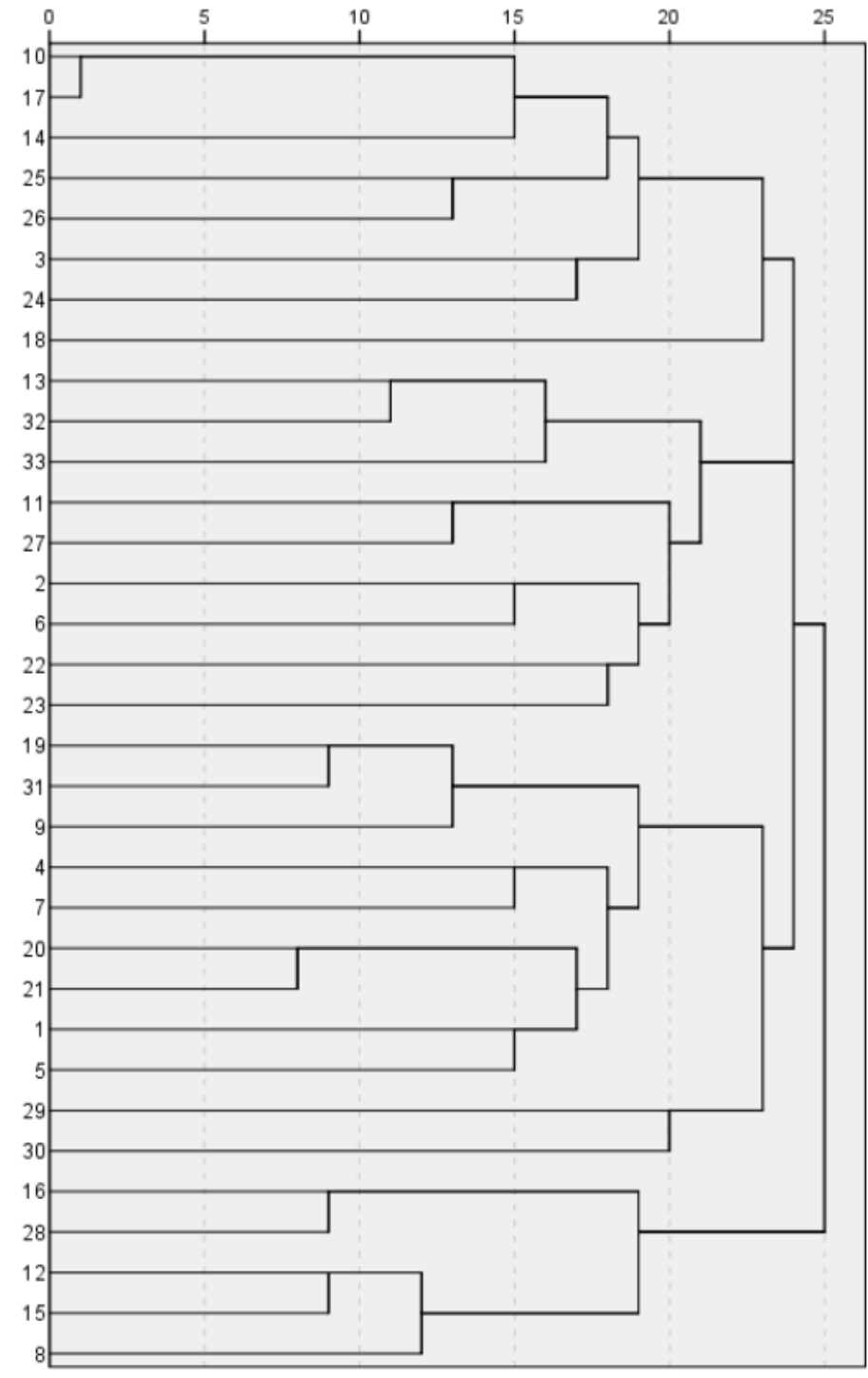

Figure 4. Drug cluster analysis tree diagram 
Table 8. Cluster analysis of drugs

\begin{tabular}{|c|c|c|c|}
\hline Cluster combination & Drug composition & Main function & Source \\
\hline $\mathrm{C} 1$ & $\begin{array}{l}\text { Safflower, peach kernel, red peony } \\
\text { root, achyranthes bidentata, lumbricus, } \\
\text { ligusticum wallichii, rhizoma corydalis }\end{array}$ & $\begin{array}{l}\text { Activating blood circulation to } \\
\text { remove blood stasis, promoting Qi } \\
\text { and relieving pain }\end{array}$ & $\begin{array}{c}\text { "Yi Lei Yuan Shu" Taohong } \\
\text { Siwu Decoction; "Medical Forest } \\
\text { Correction" Xuefu Zhuyu Decoction }\end{array}$ \\
\hline $\mathrm{C} 2$ & The root of red-rooted salvia & $\begin{array}{l}\text { Promotes blood circulation, removes } \\
\text { blood stasis, cools blood and relieves } \\
\text { trouble }\end{array}$ & - \\
\hline $\mathrm{C} 3$ & $\begin{array}{l}\text { Rehmannia glutinosa, cornus officinalis, } \\
\text { antler }\end{array}$ & $\begin{array}{l}\text { Nourishes the kidney, boosts yang, } \\
\text { nourishes the essence and marrow }\end{array}$ & - \\
\hline $\mathrm{C} 4$ & $\begin{array}{l}\text { Atractylodes atractylodes, codonopsis } \\
\text { pilosula, angelica membranaceus, radix } \\
\text { rehmanniae radix }\end{array}$ & $\begin{array}{l}\text { Invigorating spleen-stomach and } \\
\text { replenishing Qi, invigorating splenic } \\
\text { yang, raising the sinking Qi }\end{array}$ & $\begin{array}{l}\text { Buzhong Yiqi Tang in "Treatise on } \\
\text { the spleen and stomach" }\end{array}$ \\
\hline $\mathrm{C} 5$ & $\begin{array}{c}\text { Radix sileris, radix angelicae pubescentis, } \\
\text { notopterygium root, pueraria lobata root, } \\
\text { cassia twig, jujube, ginger, licorice, white } \\
\text { peony root }\end{array}$ & $\begin{array}{l}\text { Dispelling cold and dispelling wind, } \\
\text { eliminating dampness and relieving } \\
\text { pain }\end{array}$ & $\begin{array}{l}\text { Ramuli cinnamomi decoction } \\
\text { added with puerariae in "Treatise } \\
\text { on Febrile Diseases"; decoction } \\
\text { of notopterygium for expelling } \\
\text { dampness in "Treatise on the spleen } \\
\text { and stomach" }\end{array}$ \\
\hline C6 & Caulis spatholobi, Chinese clematis root & $\begin{array}{l}\text { Dispel wind and dampness, relax } \\
\text { muscles and activate collaterals }\end{array}$ & - \\
\hline $\mathrm{C} 7$ & $\begin{array}{c}\text { Gastrodia, uncaria, tangerine peel, pinellia, } \\
\text { poria }\end{array}$ & $\begin{array}{c}\text { Regulating Qi and resolving phlegm, } \\
\text { smoothing liver and extinguishing } \\
\text { wind }\end{array}$ & $\begin{array}{l}\text { "Taiping Huimin Mixture Jufang" } \\
\text { Erchen Decoction; "New Meanings } \\
\text { of Treatment of Miscellaneous } \\
\text { Diseases in Internal Medicine" } \\
\text { Tianma Gouteng Decoction }\end{array}$ \\
\hline
\end{tabular}

\subsection{Statistical results of drug association rules analysis with frequency $\geq \mathbf{2 0}$}

First, set the minimum support degree of 0.10 , the minimum confidence degree of 0.80 , and the maximum number of antecedents to 1 , to get 10 drug pair associations, see Table 9 . Once again set the minimum support degree of 0.10 , the minimum confidence degree of 0.90 , and the maximum number of antecedents to 5,27 three drug associations are obtained, see Table 10; four drug groups are related to 21 items, see Table 11; five drug groups are related to 8 items, See Table 12. In the drug association rules, the higher support groups are peach kernel-safflower, jujube-licorice, ginger-licorice, that is, blood-activating and stasis-removing medicine pair, Qi medicine pair and divergent wind-cold medicine-qi-tonifying medicine pair. In the medicine group, the higher confidence levels are ginger-cassia twig-pueraria lobata, fangfeng-pueraria lobata-licorice, peach kernel-angelica-safflower, peach kernel-licorice-safflower, radix rehmannia-radix-angelica, rehmannia glutinosa-chuanxiong-angelica, Peach kernel-Chuanxiong-Angelica-safflower, Guizhi-Pueraria lobata-Radix Paeoniae Rubra-Chuanxiong-Licorice, Guizhi-Pueraria lobata-Radix Paeoniae Rubra-Angelica-Licorice; the medicine group with a degree of elevation greater than 4 all have a pair of peach kernel-safflower, and The promotion of all rules is $>1$, indicating that there is a correlation between the items before and after each rule.

Table 9. Drug pair association

\begin{tabular}{|c|c|c|c|c|c|}
\hline The latter term & The former term & $\begin{array}{c}\text { Frequency of } \\
\text { occurrence }\end{array}$ & Support degree & Degree of confidence & Upgrading degree \\
\hline Safflower & Peach kernel & 38 & 19.10 & 94.74 & 4.10 \\
\hline Licorice & Jujube & 34 & 17.09 & 91.18 & 1.46 \\
\hline Licorice & Ginger & 33 & 16.58 & 90.91 & 1.46 \\
\hline Licorice & Bupleurum & 25 & 12.56 & 88.0 & 1.41 \\
\hline Angelica & Rehmannia & 28 & 14.07 & 85.71 & 1.48 \\
\hline Angelica & Antler & 20 & 10.05 & 85.0 & 1.47 \\
\hline Angelica & Earthworm & 24 & 12.06 & 83.33 & 1.44 \\
\hline Angelica & Codonopsis & 23 & 11.56 & 82.61 & 1.43 \\
\hline Pueraria & Radix clematidis & 21 & 10.55 & 80.95 & 1.70 \\
\hline Ligusticum wallichii & Rhizoma Corydalis & 25 & 12.56 & 80.0 & 1.56 \\
\hline
\end{tabular}


Table 10. Three associations of the drug group

\begin{tabular}{|c|c|c|c|c|c|}
\hline The latter term & The former terms & $\begin{array}{c}\text { Frequency of } \\
\text { occurrence }\end{array}$ & $\begin{array}{c}\text { Support } \\
\text { degree }\end{array}$ & $\begin{array}{l}\text { Degree of } \\
\text { confidence }\end{array}$ & $\begin{array}{c}\text { Upgrading } \\
\text { degree }\end{array}$ \\
\hline Pueraria lobata & Ginger, cassia twig & 20 & 10.05 & 100.0 & 2.10 \\
\hline Licorice & Radix sileris, pueraria lobata & 21 & 10.55 & 100.0 & 1.61 \\
\hline Safflower & Peach kernel, angelica & 28 & 14.07 & 100.0 & 4.33 \\
\hline Safflower & Peach kernel, licorice & 26 & 13.07 & 100.0 & 4.33 \\
\hline Angelica & Rehmannia, astragalus & 20 & 10.05 & 100.0 & 1.73 \\
\hline Angelica & Rehmannia, ligusticum wallichii & 21 & 10.55 & 100.0 & 1.73 \\
\hline Safflower & Peach kernel, ligusticum wallichii & 28 & 14.07 & 96.43 & 4.17 \\
\hline Licorice & Poria, qhite peony & 24 & 12.06 & 95.83 & 1.54 \\
\hline Licorice & Radix sileris, cassia twig & 21 & 10.55 & 95.24 & 1.53 \\
\hline Safflower & Peach kernel, pueraria lobata & 21 & 10.55 & 95.24 & 4.12 \\
\hline Peach kernel & Safflower, pueraria lobata & 21 & 10.55 & 95.24 & 4.99 \\
\hline Licorice & Atractylodes, white peony & 21 & 10.55 & 95.24 & 1.53 \\
\hline Pueraria Lobata & Notopterygium root, white peony & 29 & 14.57 & 93.10 & 1.95 \\
\hline Peach kernel & Safflower, licorice & 28 & 14.07 & 92.86 & 4.86 \\
\hline Licorice & Jujube, white peony & 26 & 13.07 & 92.31 & 1.48 \\
\hline Pueraria Lobata & Jujube, cassia twig & 24 & 12.06 & 91.67 & 1.92 \\
\hline White peony & Jujube, cassia twig & 24 & 12.06 & 91.67 & 1.96 \\
\hline Licorice & Jujube, cassia twig & 24 & 12.06 & 91.67 & 1.47 \\
\hline Cassia twig & Ginger, pueraria lobata & 22 & 11.06 & 90.91 & 2.55 \\
\hline Licorice & Ginger, pueraria lobata & 22 & 11.06 & 90.91 & 1.46 \\
\hline Licorice & Ginger, white peony & 22 & 11.06 & 90.91 & 1.46 \\
\hline Licorice & Radix sileris, white peony & 22 & 11.06 & 90.91 & 1.46 \\
\hline Licorice & Ginger, jujube, & 21 & 10.55 & 90.48 & 1.45 \\
\hline Licorice & Red peony, white peony & 21 & 10.55 & 90.48 & 1.45 \\
\hline White peony & Ginger, cassia twig & 20 & 10.05 & 90.0 & 1.93 \\
\hline Licorice & Ginger, cassia twig & 20 & 10.05 & 90.0 & 1.44 \\
\hline Peach kernel & Red peony, safflower & 20 & 10.05 & 90.0 & 4.71 \\
\hline
\end{tabular}

Table 11. Four associations of the drug group

\begin{tabular}{|c|c|c|c|c|c|}
\hline The latter term & The former terms & $\begin{array}{l}\text { Frequency of } \\
\text { occurrence }\end{array}$ & $\begin{array}{l}\text { Support } \\
\text { degree }\end{array}$ & $\begin{array}{l}\text { Degree of } \\
\text { confidence }\end{array}$ & $\begin{array}{c}\text { Upgrading } \\
\text { degree }\end{array}$ \\
\hline Safflower & $\begin{array}{l}\text { Peach kernel, ligusticum wallichii, angelica } \\
\text { sinensis }\end{array}$ & 21 & 10.55 & 100.0 & 4.33 \\
\hline Cassia twig & Jujube, pueraria lobata, white peony & 21 & 10.55 & 95.24 & 2.67 \\
\hline Licorice & Cassia twig, pueraria lobata, white peony & 35 & 17.59 & 94.29 & 1.51 \\
\hline Licorice & Cassia twig, white peony, ligusticum wallichii & 28 & 14.07 & 92.86 & 1.49 \\
\hline Pueraria Lobata & Notopterygium root, white peony, licorice & 24 & 12.06 & 91.67 & 1.92 \\
\hline Licorice & $\begin{array}{c}\text { Radix astragali, white peony, ligusticum } \\
\text { wallichii }\end{array}$ & 24 & 12.06 & 91.67 & 1.47 \\
\hline White peony & Jujube, cassia twig, pueraria lobata & 22 & 11.06 & 90.91 & 1.95 \\
\hline Pueraria Lobata & Jujube, cassia twig, white peony & 22 & 11.06 & 90.91 & 1.90 \\
\hline
\end{tabular}




\begin{tabular}{|c|c|c|c|c|c|}
\hline The latter term & The former terms & $\begin{array}{l}\text { Frequency of } \\
\text { occurrence }\end{array}$ & $\begin{array}{l}\text { Support } \\
\text { degree }\end{array}$ & $\begin{array}{l}\text { Degree of } \\
\text { confidence }\end{array}$ & $\begin{array}{c}\text { Upgrading } \\
\text { degree }\end{array}$ \\
\hline Licorice & Jujube, cassia twig, pueraria lobata & 22 & 11.06 & 90.91 & 1.46 \\
\hline Pueraria Lobata & Jujube, cassia twig, licorice & 22 & 11.06 & 90.91 & 1.90 \\
\hline Cassia twig & Jujube, pueraria lobata, licorice & 22 & 11.06 & 90.91 & 2.55 \\
\hline Licorice & Jujube, cassia twig, white peony & 22 & 11.06 & 90.91 & 1.46 \\
\hline White peony & Jujube, cassia twig, licorice & 22 & 11.06 & 90.91 & 1.95 \\
\hline Licorice & Jujube, pueraria lobata, white peony & 21 & 10.55 & 90.48 & 1.45 \\
\hline Peach kernel & Safflower, ligusticum wallichii, licorice & 21 & 10.55 & 90.48 & 4.74 \\
\hline Pueraria Lobata & Notopterygium root, cassia twig, licorice & 21 & 10.55 & 90.48 & 1.90 \\
\hline White peony & Ginger, cassia twig, pueraria lobata & 20 & 10.05 & 90.0 & 1.93 \\
\hline Licorice & Ginger, cassia twig, pueraria lobata & 20 & 10.05 & 90.0 & 1.44 \\
\hline Cassia twig & Ginger, Pueraria lobata, licorice & 20 & 10.05 & 90.0 & 2.52 \\
\hline Pueraria Lobata & Radix sileris, cassia twig, licorice & 20 & 10.05 & 90.0 & 1.89 \\
\hline Pueraria Lobata & Radix sileris, white peony, licorice & 20 & 10.05 & 90.0 & 1.89 \\
\hline Peach kernel & Safflower, angelica sinensis, licorice & 20 & 10.95 & 90.0 & 4.71 \\
\hline
\end{tabular}

Table 12. Five associations of drug group

\begin{tabular}{|c|c|c|c|c|c|}
\hline The latter term & The former terms & $\begin{array}{c}\text { Frequency of } \\
\text { occurrence }\end{array}$ & $\begin{array}{c}\text { Support } \\
\text { degree }\end{array}$ & $\begin{array}{l}\text { Degree of } \\
\text { confidence }\end{array}$ & $\begin{array}{l}\text { Upgrading } \\
\text { degree }\end{array}$ \\
\hline Licorice & $\begin{array}{l}\text { Cassia twig, pueraria lobata, white peony, } \\
\text { ligusticum wallichii }\end{array}$ & 22 & 11.06 & 100.0 & 1.61 \\
\hline Licorice & $\begin{array}{l}\text { Cassia twig, pueraria lobata, white peony, } \\
\text { angelica sinensis }\end{array}$ & 20 & 10.05 & 100.0 & 1.61 \\
\hline Licorice & $\begin{array}{c}\text { Pueraria lobata, white peony, ligusticum } \\
\text { wallichii, angelica sinensis }\end{array}$ & 20 & 10.05 & 95.0 & 1.53 \\
\hline Licorice & $\begin{array}{c}\text { Cassia twig, white peony, ligusticum } \\
\text { wallichii, angelica sinensis }\end{array}$ & 21 & 10.55 & 90.48 & 1.45 \\
\hline Licorice & $\begin{array}{l}\text { Jujube, cassia twig, pueraria lobata, white } \\
\text { peony }\end{array}$ & 20 & 10.05 & 90.0 & 1.44 \\
\hline White peony & Jujube, cassia twig, pueraria lobata, licorice & 20 & 10.05 & 90.0 & 1.93 \\
\hline Pueraria Lobata & Jujube, cassia twig, white peony, licorice & 20 & 10.05 & 90.0 & 1.89 \\
\hline Licorice & $\begin{array}{l}\text { Radix astragali, white peony, ligusticum } \\
\text { wallichii, angelica sinensis }\end{array}$ & 20 & 10.05 & 90.0 & 1.44 \\
\hline
\end{tabular}

\subsection{The analysis results of complex network of drugs with frequency $\geq 20$}

Set the maximum number of links to 80 , the number of links $<15$ is defined as weak links, and the number of links $>35$ is defined as strong links, and a complex network is carried out for the treatment of cervical spondylosis drugs by masters of traditional Chinese medicine and famous Chinese medicine. Analysis shows that Achyranthes bidentata, Corydalis, Cassia twig, peach kernel, Rehmannia glutinosa, licorice, ginger, atractylodes, safflower, Qianghuo, Poria, red peony, Uncaria, parsnip, tangerine peel, spatholobi, deer antler, astragalus, codonopsis, There are strong links between Pinellia, Dilong, Jujube, and Pinellia, as shown in Figure 5. 


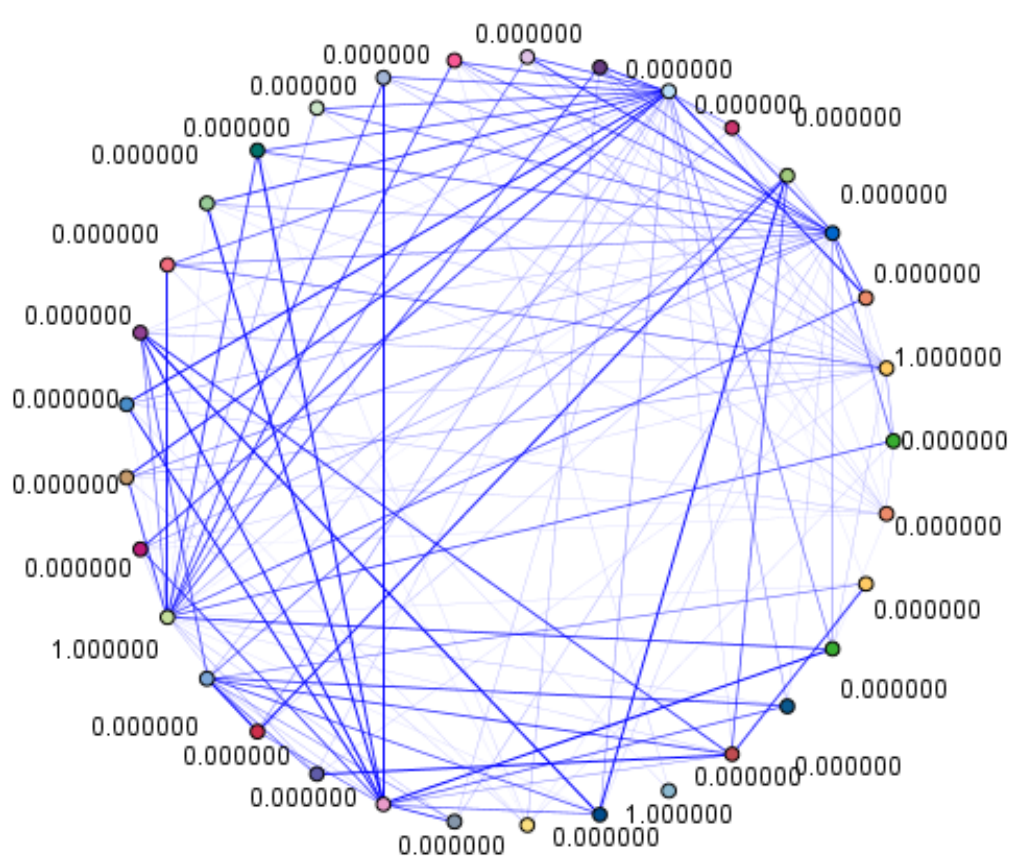

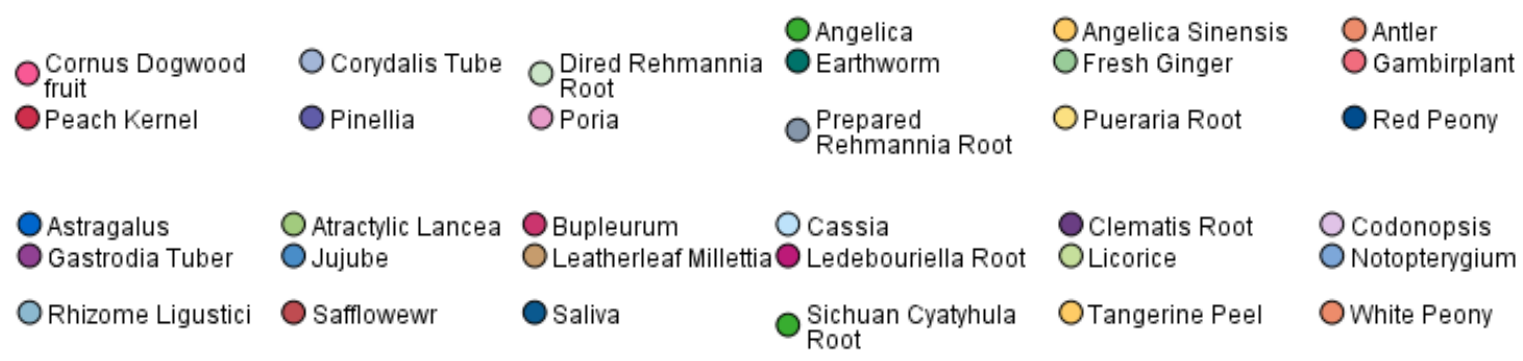

Figure 5. The complex network of drugs for the treatment of cervical spondylosis

\section{Discussion}

The clinical manifestations of cervical spondylosis are mainly pain in the neck area, accompanied by dizziness, numbness, fatigue and other symptoms. There is no such disease name in Chinese medicine. The related discussion is scattered in "Bizheng", "Fall", "Headache", "Dizziness", "Items" "Strong" and other diseases ${ }^{[1]}$ are common diseases in orthopedics and traumatology. The treatment principle of Chinese medicine for cervical spondylosis is syndrome differentiation and treatment. The treatment method is a data collection that integrates theory, law, prescription and medicine. The compatibility of prescriptions is embodied in prescription-prescription, prescription-medicine, medicine-medicine, medicine-dose, and The intricate correlation and correspondence between prescriptions and syndromes, diseases, and symptoms ${ }^{[12]}$. The prescription of traditional Chinese medicine pays attention to the compatibility of emperor, minister, adjuvant, and emissary, while traditional Chinese medicine has the difference of ups and downs, four Qi and five flavors, and return to the meridian. It is the treatment of cervical spondylosis. The types, preparation methods, dosages, and usages of Chinese medicines are ever-changing, but behind this massive, vague, seemingly random drug combination, there are clinically useful information and laws behind it. Masters of traditional Chinese medicine and famous Chinese medicine doctors have a large reserve of theoretical knowledge of traditional Chinese medicine and enrich the accumulation of clinical experience. This research is dedicated to discovering the rules of prescriptions and prescriptions of traditional Chinese medicine masters and famous Chinese medicine doctors, which can further inherit the academic thoughts and experience of famous Chinese medicine doctors, and further guide clinical practice Certificate medication.

\subsection{Frequency analysis}

The top 4 medicines are licorice, angelica, chuanxiong, and kudzu root. Angelica sinensis is sweet, warm and moisturizing, and is the holy medicine for blood. "Shen Nong's Materia Medica" records that Angelica has the effects of 
nourishing blood, activating blood, dispelling cold and relieving pain. It enters the liver and heart meridian, which coincides with the characteristics of "All winds and dizziness belong to the liver" in "Su Wen. Zhi Zhen Yao Da Lun". Chuanxiong is also blood. The medicine of Chinese Qi can "ascend the boss, descend the sea of blood, open the stagnation, bypass the meridians" and enter the liver and pericardium meridians. Angelica-Ligusticum chuanxiong medicine can nourish the blood and promote the Qi in the blood, smoothly and smoothly through the tunnel. Radix Pueraria lobata has the effect of relieving muscles and reducing fever and activating meridians. It can not only relieve fever due to the release of Xin San, but also alleviate the effects of external pathogenic depression, unfavorable meridians, and loss of nourishment of the meridians. Modern pharmacology found that: Pueraria lobata total flavonoids and Pueraria lobata isoflavones and other substances can reduce cerebrovascular resistance, vasodilation, and smooth muscle [13-14], thereby reducing peripheral resistance and alleviating the symptoms of "neck tightness". High-frequency drugs are mainly tonic drugs, activating blood and promoting Qi drugs, followed by dispelling wind, cold and dampness drugs, and dispelling wind and antispasmodic drugs. The pathogenesis of cervical spondylosis is deficiency and blood stasis as the target, and the third is to regain the sense of wind, cold and dampness. The evil corresponds. From the point of view of nature and flavor, the medicinal properties are mainly warm and flat, and there are few cold medicines, and there is no such thing as great cold and heat. The medicinal taste is most sweet, followed by bitter and pungent. Gan can nourish and relieve energy, bitterness can relieve dryness, and Xinneng San can work. It has a good effect on deficiency syndrome, cold syndrome, dampness syndrome, and phlegm syndrome.

From the statistical analysis of syndrome types and frequency of treatment methods, it can be found that pathological factors such as stagnation of qi, blood stasis, wind-cold dampness, deficiency of both Qi and blood, deficiency of liver and kidney, phlegm and dampness cannot be ignored in the onset of cervical spondylosis. If Qi stagnation and blood stasis are obvious, use products for regulating Qi and promoting blood circulation, such as peach kernels, safflower, red peony root, chuanxiong, etc.; for those with obvious cold proof, add cassia twig, kudzu root, Fangfeng and other products for dispelling wind and cold; If the phlegm-dampness stays in the central cavity, and the clear Yang does not rise and causes dizziness or loose stools, add Pinellia, Poria, Tangerine peel and other phlegm-reducing drugs; if the course of the disease is long, the liver and kidneys are damaged, then velvet antler, dogwood, and rehmannia are added. Huang and other medicines for nourishing liver and kidney, filling essence and nourishing marrow.

\subsection{Cluster analysis}

From the cluster analysis, it can be seen that 7 types of drug combinations (C1, C2, C3, C4, C5, C6, C7): C1 is the blood-activating and qi-promoting drug group, and both safflower and peach kernel are added to the blood. The power of blood stasis; Chuanxiong and Yanhusuo promote blood circulation, promote Qi and relieve pain, treat both Qi and blood at the same time; red peony root tastes bitter, clears heat and cools blood, dissipates blood stasis and relieves pain; The earth dragon tastes salty, clearing heat and stimulating the collaterals. The combination is similar to Taohong Siwu Decoction in Yili Yuanshu and Xuefu Zhuyu Decoction in Yilin Gaicuo, both of which are commonly used prescriptions for the treatment of blood stasis. A randomized controlled trial ${ }^{[15]}$ showed that the addition of Xuefu Zhuyu Decoction on the basis of conventional western medicine treatment can improve the clinical symptoms of patients and enhance the efficacy. Lu Juhong ${ }^{[16]}$ used Taohong Siwu Decoction to treat 80 cases of cervical spondylosis, but 1 case was invalid. This shows that Xuefu Zhuyu Decoction and Taohong Siwu Decoction play an important role in the treatment of patients with cervical spondylosis. C2 Danshen has a bitter taste, promotes blood circulation and removes blood stasis, cools blood to remove troubles, stays in the blood stasis, and reduces heat over time. At this time, most patients have symptoms of upset and dry mouth. Therefore, Danshen is used to clear heat and cool blood to eliminate irritability. The C3 drug group focuses on replenishing the liver and kidney, and is mostly used for severe cervical spondylosis that causes Qi stagnation and blood stasis for a long time and damages the liver and kidney. C4 constitutes Buzhong Yiqi Decoction. Related research ${ }^{[17]}$ shows that Buzhong Yiqi Decoction can effectively relax cerebral blood vessels, improve cerebral blood circulation, and relieve the symptoms of vertigo caused by cervical spondylosis. C5 contains Guizhi plus Gegen Decoction, which is commonly used in the treatment of cervical spondylosis. Qianghuo and Alone are all painful, and the two are often used in combination. C6 has the effect of dispelling dampness and promoting blood circulation. C7 tangerine peel, pinellia, and Poria have the embryonic form of Erchen Decoction, and Gastrodia and Uncaria have the embryonic form of Gastrodia and Uncaria. This medicine group eliminates wind and resolves phlegm, and can be used for the symptoms of cervical spondylosis caused by wind phlegm disturbance. From the results of cluster analysis, it can be seen that masters of traditional Chinese medicine and famous Chinese doctors believe that the pathological factors of cervical spondylosis are deficiency, blood stasis, wind, cold, dampness, and phlegm. Wetness, phlegm and collaterals. 


\subsection{Analysis of association rules}

The commonly used drug combinations for the treatment of cervical spondylosis are mostly different combinations of drugs for promoting blood circulation, promoting qi, replenishing qi, replenishing blood, relieving the surface, removing dampness, diuresis, and cooling blood. Peach kernel-safflower has the highest degree of support. Peach kernels are bitter and sweet in nature, blood-breaking, stagnant and moisturizing; safflower has a pungent taste, promotes blood circulation, removes blood stasis and relieves pain. Professor Huang Dehong ${ }^{[18]}$ believes that the medicine has the meaning of nourishing blood in promoting blood circulation, can invigorate blood without hurting righteousness, and has a good effect on cervical spondylosis with stagnation of Qi and blood stasis. The nine groups with higher confidence are Guizhi-pueraria lobata root, peach kernel-safflower, radix rehmanniae-angelica drug pair, cassia twig anti-muscle release, and Pueraria lobata root enhances the anti-muscle release power; Rehmannia glutinosa has a warm and sweet taste, nourishes yin and blood, Angelica sinensis is sweet and warm, and has the effect of activating blood. The two medicines are matched, the movement and the static are suitable, the blood is not stagnated, and the blood is not damaged. Peach kernel-safflower plus angelica, licorice, pueraria lobata, red peony root, the elevation is greater than 4: Angelica tonifies blood and promotes blood circulation, licorice nourishes the spleen and qi, pueraria lobata relieves muscles and reduces fever, relieves meridians and collaterals, red peony clears heat and cools blood, relieves blood stasis and relieves pain, Corresponding to the main pathological factors of cervical spondylosis, blood, qi, deficiency, cold, and blood stasis ${ }^{[19]}$, clinical reference can be provided for addition and subtraction compatibility. The results of the association rule analysis show that: in the treatment of cervical spondylosis, famous and old Chinese medicine doctors mostly treat cervical spondylosis with Qi and blood, which can invigorate Qi and blood while promoting blood circulation, promoting Qi and dispelling cold.

\subsection{Complex network analysis}

The core combination for the treatment of cervical spondylosis is mainly based on activating blood and promoting Qi medicine, Qi and nourishing medicine, phlegm and dampness medicine, and dispelling wind and cold medicine, indicating that the famous traditional Chinese medicine treatment of cervical spondylosis pays attention to nourishing Qi and blood, taking into account phlegm and dampness, and expelling wind. Dispel the cold. It shows that the disease is located in the Qi in the blood, Qi and blood are the root, and pathological products such as blood stasis, wind, cold, dampness, and phlegm are the targets. The course of the disease is prolonged and the liver and kidneys are damaged. Clinically, rehmannia glutinosa and dogwood should be added to nourish the liver and kidney. While replenishing the liver and kidney, it is added with Caulis spatholobi, Dilong and other drugs to relax the tendons and activate the collaterals, so that it can nourish but not stagnate. This disease is based on stagnation of Qi and blood stasis, and the three evils of wind, cold and dampness are exogenous, and cassia twig, Qianghuo, and wind-proof medicine are added. Guizhi sweats and relieves muscles, activates Qiang, prevents wind and dispels cold, overcomes dampness and relieves pain. However, this kind of drugs has strong resolving power, and the dosage should not be too strong. It is often compatible with blood and Qi drugs to eliminate evil and not harm the body.

To sum up, the well-known traditional Chinese medicine treatment of cervical spondylosis mostly uses activating blood to remove blood stasis, replenishing the liver and kidney, and the drugs are mostly warming and sweet, returning to the spleen and liver meridian. On the basis of activating blood and removing blood stasis, it also takes into account the movement of Qi and the basis of dispelling wind. On the basis of collaterals, removing dampness on the basis of resolving phlegm, and dredging on the basis of replenishing liver and kidney. This research has largely avoided the biases of individual physicians in the diagnosis and treatment of symptoms and signs, and it also objectively reflects the general rules of modern Chinese medicine for the treatment of cervical spondylosis and the general rules of prescriptions and medications. This study uses data mining methods to explore the rules of prescriptions of famous and old Chinese medicine practitioners in the treatment of cervical spondylosis. Because the dosage of prescription drugs is also very important, the prescriptions and drugs are the result of syndrome differentiation and treatment. The article analyzes the types of syndromes. However, the syndrome type is the result of the four diagnoses, so the statistical analysis of the four diagnoses can dig out the symptomdrug relationship, which is not introduced due to limited space. To a certain extent, the article introduces the prescription of famous traditional Chinese medicine doctors in the treatment of cervical spondylosis, and provides guidance for the clinical use of cervical spondylosis.

\section{Acknowledgments}

This research was supported by the following fund projects: the 2018 "New Development Project" scientific research project of the First Affiliated Hospital of Tianjin University of Traditional Chinese Medicine (201908). 


\section{References}

[1] Liang Long, Feng Minshan, Yinhe, Wei Xu, Gao Chunyu, Yin Xunlu, Zhu Liguo. Application of functional exercise in the treatment of radiculopathy of cervical spondylosis. Western Traditional Chinese Medicine. 2020; 33(03): $107-110$.

[2] Li Hongguang, Sun Shiyi, Zhu Xiaoyan. The pathological changes of cervical spondylosis. Journal of Jilin Medical College. 2010; 31(06): 338-339.

[3] Zhu Tingyan, Duan Yaping, Wu Xiaoyong, Yang Shimei. Progress in traditional Chinese medicine treatment of cervical spondylosis. Journal of Guiyang College of Traditional Chinese Medicine. 2018; 40(05): 90-92.

[4] Wu Zhihai, Wu Yangjian, Wang Kun. Meta-analysis on the treatment of cervical spondylosis with Chinese medicine guidance. Massage and Rehabilitation Medicine. 2021; 12(03): 48-51+57.

[5] Xu Jinhai, Yang Aimin, Shi Wenmin, Wang Jing, Wang Guodong, Zhou Xiaoning, Kuang Yulin, Qiao Jiaojiao, Ye Jie, Mo Wen, Shi Qi. Effectiveness and safety of Chinese medicines for promoting blood circulation and removing blood stasis in the treatment of cervical spondylotic radiculopathy Systematic review of Chinese medicine. Traditional Chinese Medicine Orthopedics. 2019; 31(12): 20-28.

[6] Shen Yi, Fu Ping, Kong Liya. Application of data mining method in the research of famous and old traditional Chinese medicine drugs. Journal of Traditional Chinese Medicine. 2016; 57(10): 890-893.

[7] Zhong Gansheng. Chinese Materia Medica. Beijing: China Press of Traditional Chinese Medicine; 2012.

[8] National Pharmacopoeia Committee. The People's Republic of China Pharmacopoeia (2015 edition). Beijing: China Pharmaceutical Science and Technology Press; 2015.

[9] Zhu Wenfeng. Diagnostics of Traditional Chinese Medicine. Beijing: China Press of Traditional Chinese Medicine; 2006

[10] Ma Yong. Traditional Chinese Medicine Muscle Injury. Beijing: People's Medical Publishing House; 2012.

[11] Wang Heming, Huang Guicheng. Traditional Chinese Medicine Orthopedics. Beijing: China Press of Traditional Chinese Medicine; 2012.

[12] Yang Yuzhu. Summary and Application of Data Mining Technology. Henan Science and Technology. $2014 ; 10$ (19): 21.

[13] Zhang Junxia. Analysis of the effect of puerarin in the treatment of cerebral infarction. Contemporary Medical Essays. 2017; 15(02): 143-144.

[14] Chu Jiming, Ma Shuyun, Li Haifeng, et al. Research progress on the effective ingredients and pharmacological effects of Pueraria lobata. Food and Drugs. 2015; 17(02): 142-146.

[15] Li Bin. Xuefu Zhuyu Decoction Treats 48 Cases of Cervical Spondylopathy with Qi Stagnation and Blood Stasis Syndrome. Herald of Traditional Chinese Medicine. 2012; 18(12): 105.

[16] Lu Juhong. Analysis of the curative effect of Jiawei Taohong Siwu Decoction in the treatment of vertebral artery type cervical spondylosis. Chinese Community Physician (Medical Specialty Semi-Monthly). 2008; (20): 126-127.

[17] Xing Qiujuan, Zhao Dongfeng, Wu Ji, et al. Study on the efficacy of Yiqihuoxuefang combined with betahistine mesylate in the treatment of vertebral artery type cervical spondylopathy (insufficiency of Qi and blood). Chinese Emergency in Traditional Chinese Medicine. 2019; 28(7):1201-1204.

[18] Xu Xingyi, Chen Xiuhui, Huang Dehong. Professor Huang Dehong's experience in clinical application of Taohong Siwu Decoction. Chinese Materia Medica and Clinic. 2017; 8(5): 33-34.

[19] Zhou Xueping, Wu Mianhua. Internal Medicine of Traditional Chinese Medicine. Beijing: China Press of Traditional Chinese Medicine; 2017. 